\title{
Auditory functional magnetic resonance imaging in dogs - normalization and group analysis and the processing of pitch in the canine auditory pathways
}

\author{
Jan-Peter Bach ${ }^{1 *}$ (D) Matthias Lüpke ${ }^{2}$, Peter Dziallas ${ }^{1}$, Patrick Wefstaedt ${ }^{1}$, Stefan Uppenkamp ${ }^{3}$, Hermann Seifert $^{2}$ \\ and Ingo Nolte ${ }^{1}$
}

\begin{abstract}
Background: Functional magnetic resonance imaging (fMRI) is an advanced and frequently used technique for studying brain functions in humans and increasingly so in animals. A key element of analyzing fMRI data is group analysis, for which valid spatial normalization is a prerequisite. In the current study we applied normalization and group analysis to a dataset from an auditory functional MRI experiment in anesthetized beagles. The stimulation paradigm used in the experiment was composed of simple Gaussian noise and regular interval sounds (RIS), which included a periodicity pitch as an additional sound feature. The results from the performed group analysis were compared with those from single animal analysis. In addition to this, the data were examined for brain regions showing an increased activation associated with the perception of pitch.

Results: With the group analysis, significant activations matching the position of the right superior olivary nucleus, lateral lemniscus and internal capsule were identified, which could not be detected in the single animal analysis. In addition, a large cluster of activated voxels in the auditory cortex was found. The contrast of the RIS condition (including pitch) with Gaussian noise (no pitch) showed a significant effect in a region matching the location of the left medial geniculate nucleus.

Conclusion: By using group analysis additional activated areas along the canine auditory pathways could be identified in comparison to single animal analysis. It was possible to demonstrate a pitch-specific effect, indicating that group analysis is a suitable method for improving the results of auditory fMRI studies in dogs and extending our knowledge of canine neuroanatomy.
\end{abstract}

Keywords: fMRI, Functional magnetic resonance imaging, Auditory system, Dog, Normalization, Pitch

\section{Background}

The auditory sense plays a significant role in many aspects of a dog's life. It is important for the communication with humans and conspecifics and helps detect environmental threats such as motor vehicles [1]. An impairment of the hearing sense can severely impact a dog's ability to function in its environment. Different methods have been used to examine the auditory

\footnotetext{
* Correspondence: jbach@tiho-hannover.de

${ }^{1}$ Klinik für Kleintiere, Stiftung Tierärztliche Hochschule Hannover, Bünteweg 9, 30559 Hannover, Germany

Full list of author information is available at the end of the article
}

function in dogs [2-5], among them electroaudiometry, which has also become the most important test for the clinical evaluation of hearing in canine patients [6]. Electroaudiometry is an objective, easy to perform and minimally invasive technique for evaluating the hearing function in dogs and other mammals [7]. Still, conventional electroaudiometry, which assesses the hearing function through electrodes placed subcutaneously around the skull, provides only very limited information about the spatial origin of the measured signals [8] and does not allow for an examination of the cortical auditory functions [9]. More detailed examinations of 
the central hearing functions with electrodiagnostic methods, as have been performed in cats, are feasible but highly invasive [10].

One of the most important techniques in hearing research in humans is functional magnetic resonance imaging (fMRI) [11]. fMRI is a noninvasive technique with a high spatial resolution capable of identifying brain regions reacting to specific stimuli presented to the subject during an fMRI-scanning-session. To do this, fMRI utilizes the BOLD (blood oxygenation level dependent) effect. The BOLD effect originates from an increase in metabolic activity and blood flow in activated parts of the central nervous system. As the increased blood flow exceeds the increased demand for oxygen, there is an increase in the ratio of oxygenated hemoglobin to deoxygenated hemoglobin in the activated areas, which can be detected as a slight signal rise in special fMRI sequences [12]. fMRI has been used in a huge number of studies to identify the regions responsible for the perception of different auditory features in the human brain [13-15], including the perception of pitch $[16,17]$. As the auditory cortex is the location for performing higher auditory functions, these studies have highly improved our understanding of auditory perception in humans $[11,15]$ and other vertebrates $[18,19]$.

Due to the fact that it is required for the subject to remain motionless during the scanning process, it is in most cases necessary to employ anesthesia or physical restraint in fMRI experiments in animals [20-24]. Using physical restraint in awake animals is not only problematic due to ethical reasons, but in addition to this the results of such experiments may be influenced by stress or other neural activity induced by lying in the scanner. For these reasons, most fMRI experiments performed in animals use anesthesia to inhibit subject movement [20, 21, 24, 25].

Unfortunately, anesthesia strongly reduces the extent of the measured BOLD effect, making it difficult to detect neural responses via fMRI [26]. Even in awake humans, the detectable BOLD signal change is rather small and there are many factors influencing the intensity of the measured MRI signal. Therefore, it is necessary to obtain many images to distinguish signal changes elicited by stimuli from random signal changes: with sufficient numbers of repetitions, random signal changes tend to average out, while signal changes due to stimulation persist [27].

A technique frequently used in fMRI-studies in humans is normalization [28-30]. Normalization allows for the combined analysis of data across several subjects participating in a study, thereby increasing the number of images available and improving the study's statistical power [31]. In addition to this, normalization reduces the influence of intersubject variability on the measured results and allows for the comparison of the neural activity of different groups (e. g. persons suffering from a certain disease compared to healthy individuals) [30]. For the present study, anatomical images and functional scans following auditory stimulation of ten anesthetized beagle dogs were acquired using MRI. One of two stimulation paradigms used was comprised of two different kinds of auditory stimuli: 1 . simple Gaussian noise stimuli and 2. regular interval sounds (RIS) including a periodicity pitch as an additional sound feature [32]. First results of this study, including the findings of single animal analysis and the comparison of the two stimulation paradigms used, have already been published [33]. For the present article the functional data of each dog was normalized to a template image, which was developed by Datta et al. [34] to be used as a template for canine fMRI studies, and a statistical group analysis was performed. A statistical parametric map displaying the activated areas in the canine brain following auditory stimulation was generated and a region of interest (ROI)-analysis was performed. Afterwards, the results of the group analysis were compared to the results of single dog analysis. Finally, the effect of periodicity pitch on the activation in the ascending stages of the canine auditory system was examined by comparing the activation elicited by simple Gaussian noise to the activation produced by RIS. The results of these examinations were used to address the following questions: 1. Do normalization and group analysis improve the detection of activated regions along the auditory pathways in dogs? 2. Is the amount of incidental activation, which does not show any connection to the auditory pathways, reduced in the group analysis in comparison to the single animal analysis? 3. Are there regions which show an increased activation following stimulation with sounds including pitch in comparison to simple Gaussian noise?

\section{Results}

Two of the ten dogs participating in the study were used to test the experimental setup and optimize the stimulation paradigms and functional imaging sequences, resulting in the inclusion of the functional data sets of eight dogs in the final study. A comparison of all sound conditions vs. silence yielded the following results: with sequence 1, which used the sparse temporal sampling method [35], significantly activated voxels were found in the regions of interest (ROIs) defined for the caudal colliculi (CC) and the medial geniculate nuclei (MGN) in the group analysis (Fig. 1). In comparison to the single animal analysis, where each ROI showed significantly activated voxels in every dog, no activation was evident in the ROI created for the temporal cortex (TC). In group 

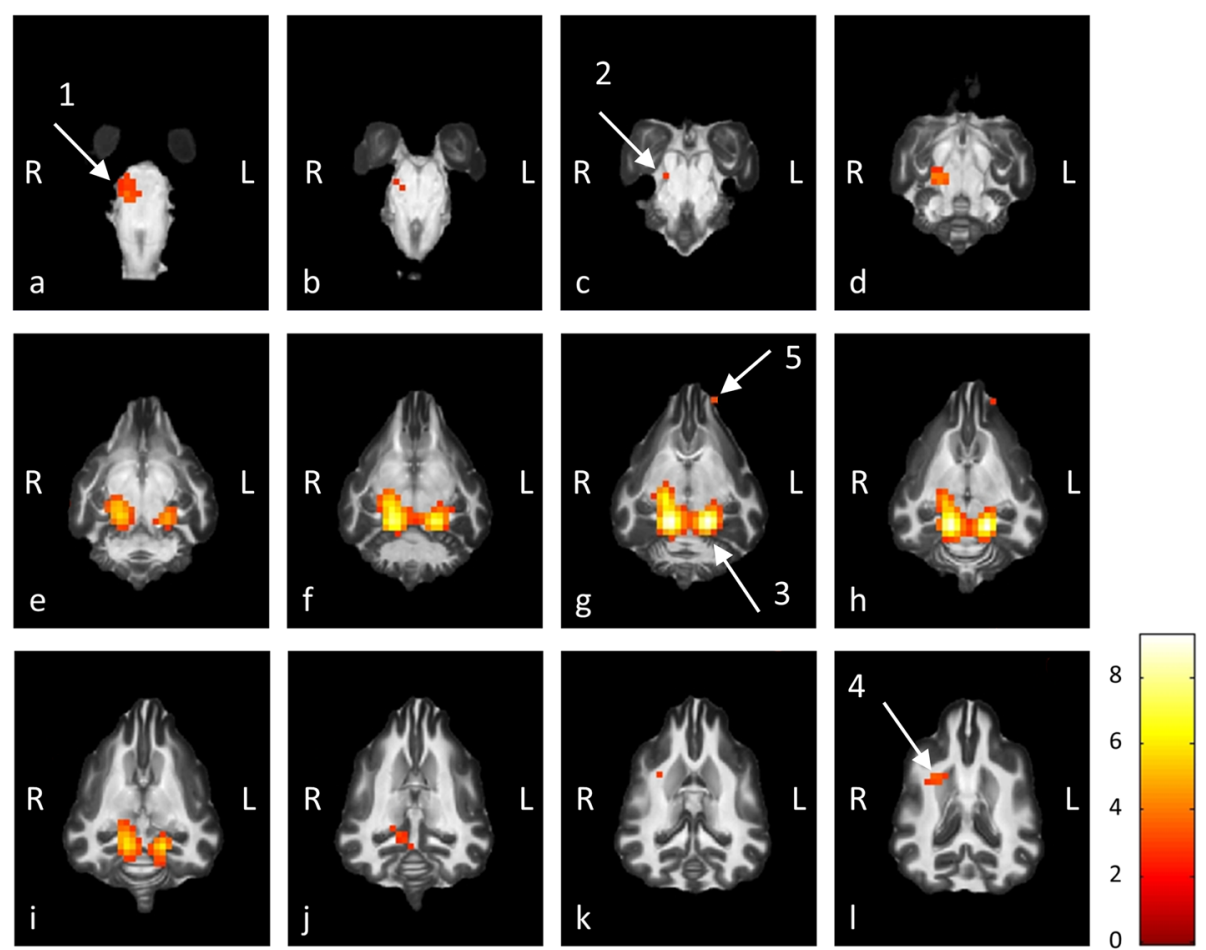

Fig. 1 Group Analysis of auditory fMRI data following binaural stimulation with sparse temporal sampling paradigm. Functional data following auditory stimulation was collected for each dog using a T2* weighted functional MRI sequence. Afterwards the data was normalized to Datta's atlas of the dog brain [34] and a statistical group analysis was performed. In the images above, all regions showing significant activation are displayed as colored pixels, which were superimposed onto dorsal slices of Datta's brain atlas [34]. The gap between two adjacent slices is $2 \mathrm{~mm}$. The colorbar indicates the $t$-values of the activated voxels. The pictures show unilateral activation of the right superior olivary nucleus in the brainstem (a-b, arrow 1). This activation can be traced dorsally along the lateral lemniscus (c-d, arrow 2) to the much larger bilateral activation of the caudal colliculi and further rostrally to the medial geniculate nuclei (e-j); merging activations marked with arrow 3 . An activation matching the position of the right internal capsule can be found on pictures $\mathbf{k - I}$ (arrow 4). In addition to this, a small activated area was found in a region which is not normally associated with the auditory system (g-h, arrow 5). No significantly activated voxels were found in the auditory cortex

analysis additional clusters of activated voxels were found matching the position of the right superior olivary nucleus, lateral lemniscus and internal capsule, which are known to have an auditory function in mammals (Figs. 1, 2) [14, 36, 37].
With sequence 2 significantly activated voxels were found in 4 dogs in the CC region, 3 dogs in the MGN region and 5 dogs in the $\mathrm{TC}$ region in the single animal analysis. The group analysis of the functional data acquired with sequence 2 showed significantly activated
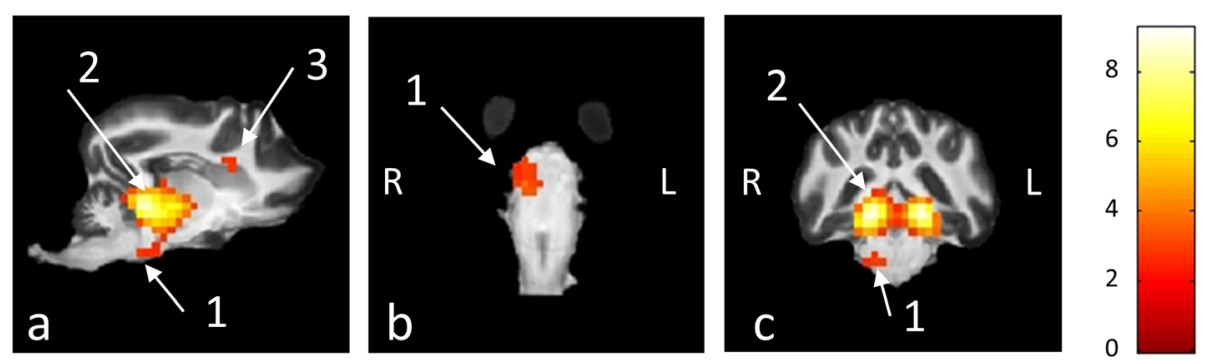

Fig. 2 Unilateral activation of the olivary nucleus and the internal capsule. The image displays the unilateral activation of the right superior olivary nucleus (arrow 1), which was found following group analysis of the functional data obtained with sequence 1. The activation is displayed in sagittal (a), dorsal (b) and transverse (c) planes. In image (a), the activation can be traced along the lateral lemniscus to the much larger activation of the right caudal colliculus (arrow 2), which is also displayed on image (c). Image a also displays the dorsal aspect of the activation of the internal capsule already seen in Fig. $1(\mathrm{k}-\mathrm{l})$. It has to be noted that the part of the activation seen here (arrow 3 ) exceeds the anatomical boundaries of the internal capsule 
voxels in all ROIs, including a large cluster of activated voxels in the left temporal cortex. The activation in the temporal cortex was located in the ectosylvian gyrus (Fig. 3), which is consistent with lesion studies in dogs $[38,39]$ and other recent fMRI studies in dogs and cats [40, 41]. Though it was not possible to detect any cortical activation with the sparse temporal sampling paradigm, this paradigm elicited considerably higher activation in the subcortical auditory pathways. The statistical values calculated for the different ROIs can be found in Table 1 . Following group analysis, $t$-values were considerably higher for all defined ROIs in comparison to the average $t$-values of the single animal analysis for the functional data obtained with sequence 2 . For the data acquired with sequence 1 , a higher t-value following group analysis was only calculated for the MGN region. The $\mathrm{t}$-value for the $\mathrm{CC}$ region was slightly reduced in comparison to the single animal analysis. The $\mathrm{t}$-value for the $\mathrm{TC}$ region, which was negative in the single animal analysis, was further reduced.

On comparing regular interval sounds (RIS) with simple Gaussian noise a significant BOLD contrast was found in the MGN region (Fig. 4). The contrast yielded no significant activation for the $\mathrm{CC}$ and $\mathrm{TC}$ regions, and again little activation was detected outside the ROIs for this contrast. The peak $t$-values for this comparison were $t=2.02$ (CCregion), $\mathrm{t}=3.27$ (MGN-region) and $\mathrm{t}=2.33$ (TC-region).
Table 1 Statistical values obtained with sequences 1 and 2 in the single animal and group analysis

\begin{tabular}{|c|c|c|c|c|c|c|}
\hline \multicolumn{7}{|c|}{ Sequence 1} \\
\hline & \multicolumn{3}{|c|}{ Single animal analysis (average) } & \multicolumn{3}{|c|}{ Group analysis } \\
\hline & $\begin{array}{l}\text { Average } \\
\text { t-value }\end{array}$ & $\begin{array}{l}\text { Signal } \\
\text { change (\%) }\end{array}$ & $\begin{array}{l}\text { Activated } \\
\text { voxels (\%) }\end{array}$ & $\begin{array}{l}\mathrm{t}- \\
\text { value }\end{array}$ & $\begin{array}{l}\text { Signal } \\
\text { change (\%) }\end{array}$ & $\begin{array}{l}\text { Activated } \\
\text { voxels (\%) }\end{array}$ \\
\hline CC & 10.04 & 0.318 & 54.77 & 9.53 & 0.245 & 88.89 \\
\hline MGN & 4.5 & 0.249 & 32.96 & 6.11 & 0.143 & 68.52 \\
\hline $\mathrm{TC}$ & -2.01 & -0.032 & 0.52 & -2.74 & -0.045 & 0.08 \\
\hline \multicolumn{7}{|c|}{ Sequence 2} \\
\hline CC & 1.93 & 0.070 & 8.24 & 3.20 & 0.051 & 7.41 \\
\hline MGN & 1.32 & 0.083 & 2.46 & 2.08 & 0.036 & 3.70 \\
\hline $\mathrm{TC}$ & 0.77 & 0.023 & 2.05 & 3.20 & 0.050 & 3.88 \\
\hline
\end{tabular}

The average t-value of all voxels included in the ROI, the mean percentage signal change between sound and silence conditions and the percentage of voxels showing significant activation are listed for the different sequences and ROIs (CC caudal colliculi, MGN medial geniculate nuclei, TC temporal cortex). The statistical values for the subcortical ROls are higher with sequence 1, whereas only sequence 2 recorded a positive t-value and signal change for the cortical ROI

\section{Discussion}

One of the aims of this study was to examine whether normalization and group analysis improve the detection of BOLD activation in the canine auditory pathway following auditory stimulation. For the subcortical ROIs, significantly activated voxels were found in all
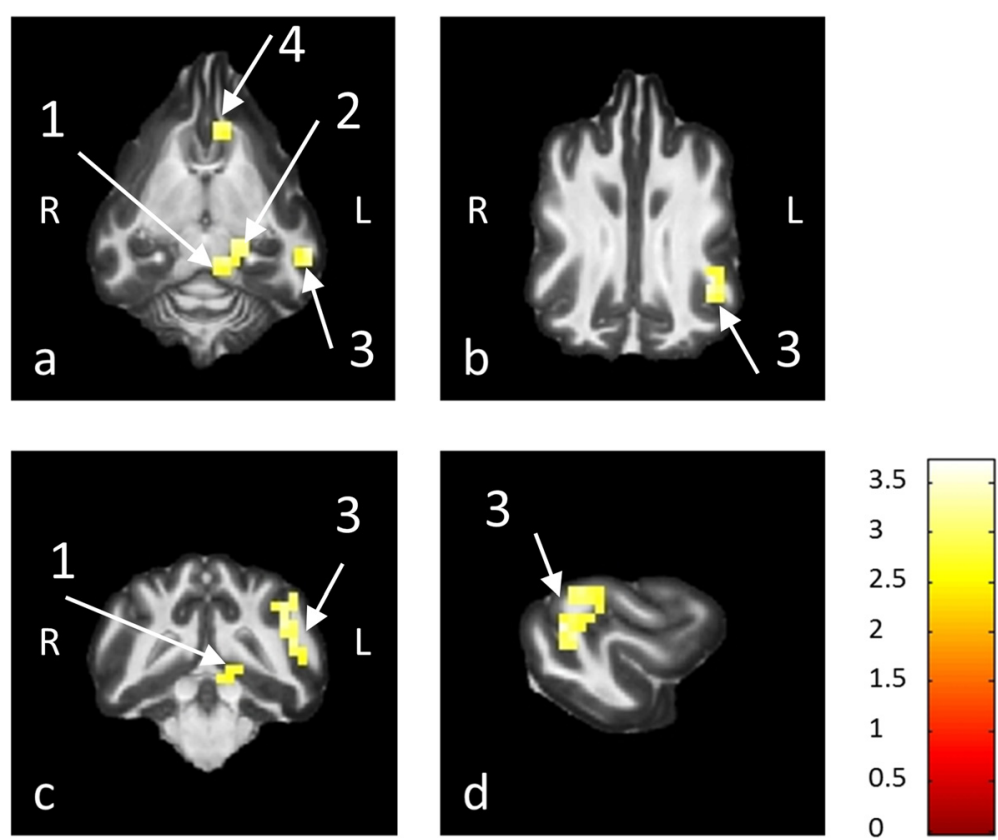

Fig. 3 Group Analysis of auditory fMRI data following stimulation with continuous scanning paradigm. The group analysis of the functional data obtained with sequence 2 in combination with paradigm 2 (continuous stimulation) yielded unilateral activation of the left caudal colliculus (arrow 1), medial geniculate nucleus (arrow 2) and gyrus ectosylvius (arrow 3), which is part of the auditory cortex. These activations are displayed in dorsal $(\mathbf{a}, \mathbf{b})$, transverse $(\mathbf{c})$ and sagittal (d) sections. In addition to these activations of the auditory pathways, an activated area is seen at the rostral aspect of the left caudate nucleus (a, arrow 4) 

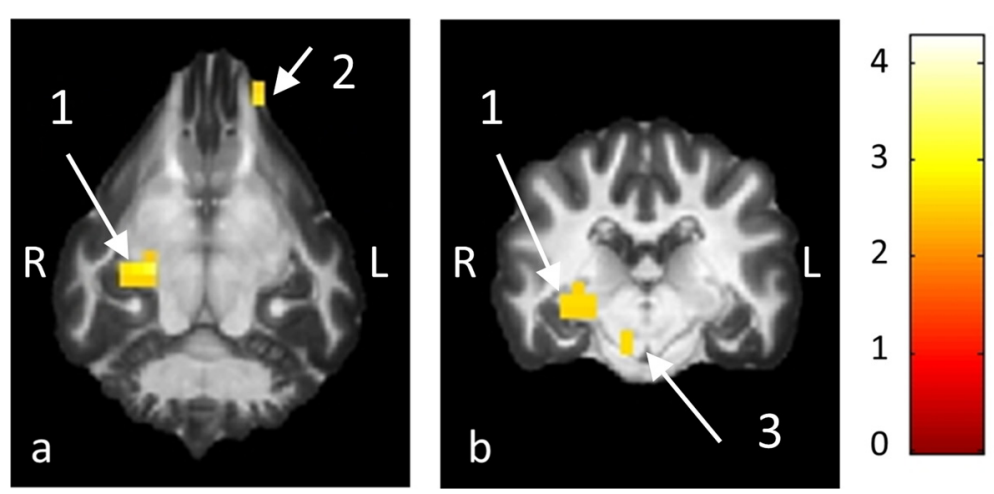

Fig. 4 Comparison of RIS with simple Gaussian noise. The comparison of RIS with simple Gaussian noise using the functional data obtained with sequence 1 showed a significant contrast for the right medial geniculate nucleus (arrow 1), which is displayed in the dorsal (a) and the transverse (b) plane. In addition to this, small activations can be seen in the images without spatial relation to any known structures of the auditory system (arrows 2, 3)

regions for both stimulation paradigms following group analysis. As the same is true for the majority of dogs in the single animal analysis, a beneficial effect of the group analysis is not clearly supported by this finding.

Concerning the existence of activated voxels in the TC $\mathrm{ROI}$ in the single animal analysis and the lack of these in the group analysis with sequence 1 , it has to be noted that the activated voxels in the single animal analysis were few and not always located in areas commonly associated with the auditory system. Thus, it seems likely that part of the activations in the TC ROI, which was comparatively large in comparison to the small ROIs defined for the subcortical parts of the auditory pathways, may have been incidental in the single animal analysis. Due to the large extent of the TC ROI it is also possible, that this region included voxels which were not part of the auditory system, thus leading to rather small average $\mathrm{t}$-values for this area in comparison to the other ROIs. The activation in the TC ROI, which was found in the group analysis following continuous stimulation however, was large and located in the ectosylvian gyrus, which was identified as part of the auditory pathway in other studies [40, 41].

In addition to the activated regions found in the single animal analysis, additional activated areas were detected with sequence 1 at the location of the internal capsule and in the brainstem matching the position of the right superior olivary nucleus [42]. Though an auditory function of these structures could be suspected due to studies in other mammals, this study is the first to confirm these assumptions in dogs via fMRI. Since it was not possible to detect a significant activation in these areas in single animal analysis, these results indicate that normalization and group analysis are suitable for facilitating the detection of activated voxels along the auditory pathway in canine fMRI-studies, especially concerning smaller signal changes, which might otherwise be missed due to the decreased BOLD contrast associated with anesthesia. The digital atlas of the dog brain developed by Datta et al. [34], though derived from the brains of mixed-breed dogs, seems to be adequate as a template for the normalization and group analysis of fMRI data acquired in studies in beagles and possibly other mesaticephalic dogs. Whether the same is true for brachycephalic and doliocephalic dogs has to be examined in future studies.

The reason why some of the activation found with sequence 1 and sequence 2 was only detected on one side, although binaural auditory stimulation was applied, remains unclear. A possible explanation could be that the stimulation applied to both ears was not perceived in the same manner. Though great effort was invested on placing the earphones in identical positions in both external ear canals and performing the stimulation at the exact same sound level, a displacement of the earphones (originally designed for use in human subjects) could have resulted in different stimulation of the ears. In addition to this, the ear protectors placed above the earphones may have been positioned slightly differently on both sides, resulting in a decreased activation following auditory stimulation due to background noise.

Very few activated voxels were found which were not located in or directly adjacent to known structures of the auditory pathway. In fMRI studies in awake people, high statistical thresholds and additional measures like family-wise-error correction are used to distinguish random signal changes from signal changes due to stimulation. As it is common to use lower statistical thresholds in animals, it is of great importance to minimize the false positive results by other means. Group analysis seems to be a suitable method for this purpose.

Another benefit of normalization and group analysis as performed for this study is the generation of a dataset which represents the neural activity found in 
a group of different subjects, thereby reducing the influence of individual abnormalities in functional neuroanatomy. The resulting dataset and the template used for normalization and group analysis are freely available, allowing other researchers to easily compare and combine the results of this study with their own findings in future studies.

Another important aspect of the current study was the investigation of the effect of pitch on the auditory perception of the dog. Pitch-specific neural activity has already been identified in humans in numerous studies $[16,32,43,44]$. In this study, the neural activation in the MGN ROI was significantly increased for stimuli including pitch as a sound feature in comparison to simple Gaussian noise. No significant difference was detected in other parts of the auditory system. Pitch is an important feature of sound that plays an eminent role in daily communication of people: The pitch of speech can inform the listener about the age, gender and emotional state of the speaker $[45,46]$. Although it is difficult to investigate whether animals perceive sound in the same manner as humans, many studies suggest that the perception of pitch plays an important role in the communication of animals as well [47-50]. Due to the many similarities in pitch perception among species, animal models may provide information about the neural processes connected with the auditory perception in different species, including humans [44]. As it is possible to train dogs to remain motionless in the MRI-scanner during fMRI-experiments, as has been shown in recent studies $[40,51,52]$, the dog model seems to be well suited for the research of the auditory system and other neural processes in animals in the future.

In addition to answering the questions asked at the beginning of this study, group analysis also provides new information concerning the suitability of the two stimulation paradigms for their use in auditory fMRI studies in dogs: while paradigm 1 seemed to be superior to paradigm 2 in the single animal analysis due to stronger activation of the subcortical auditory pathways, the considerable activation of the auditory cortex following group analysis shows that a continuous stimulation may also offer benefits. For these reasons, a general superiority of sparse temporal sampling over continuous scanning methods as indicated in various auditory fMRI studies in humans and monkeys $[25,35,53]$ cannot be stated for canine auditory fMRI. The finding of a significant activation in the auditory cortex with sequence 2 in comparison to sequence 1 , where no cortical activation was found in this study, is in accordance with a study in cats, where continuous stimulation resulted in a larger extent of cortical activation than stimulation with a sparse paradigm [41]. Hall et al. [41] did not find a significant difference between continuous und sparse stimulation for the subcortical parts of the auditory system, though.

\section{Conclusions}

Following group analysis it was possible to show a pitch-specific effect in the canine brain and detect additional activated areas along the canine auditory pathways in comparison to single animal analysis. These findings support the assumption that group analysis is a suitable method for improving the results of auditory fMRI studies in dogs. As to the comparison of the two stimulation paradigms it can be summarized that stronger activation of the subcortical auditory pathways was detected with the sparse temporal sampling paradigm, whereas significant activation in the temporal cortex could only be found following stimulation with the continuous paradigm. Altogether fMRI offers interesting opportunities for future research concerned with canine hearing disorders and neuroanatomy. Regarding possible clinical applications of auditory fMRI in animal patients, the need of anesthesia hinders a widespread use in clinical practice, as other testing procedures like electroaudiometry, though lacking the detailed spatial information given by fMRI, are easier to perform and produce more reliable results in anesthetized animal patients.

\section{Methods}

The study was designed as a prospective, experimental study.

\section{Animals}

Ten healthy beagles were included in the study. The mean age of the beagles was 3.7 years (+-2.3 SD) and they had an average body weight of $16.0 \mathrm{~kg}$ (+-2.6 SD). Prior to the examination in the fMRI scanner, a physical examination and a neurological examination were performed to rule out any hearing impairments or an increased anesthetic risk. In addition to this, an otoscopic examination and a BAER test were conducted during the same anesthesia in which the MRI examinations took place. Details of the BAER examination and the performed anesthesia can be found in [33]. All participants of the study were clinic-owned beagles from the Small Animal Clinic of the University of Veterinary Medicine Hannover. All procedures were approved by the Animal Welfare Officer of the University of Veterinary Medicine Hannover and the Lower Saxony State Office for Consumer Protection and Food Safety, Oldenburg, Germany (TV-No. 33.9-42502-0512A223). 


\section{Stimuli}

The stimuli used to elicit a BOLD response in the beagles' brains were simple random noise stimuli (Gaussian noise, i. e., the digital signal was made of normally distributed random numbers), bandpass-filtered between $250 \mathrm{~Hz}$ and $4 \mathrm{kHz}$, and regular interval sounds (RIS), generated from the random noise by an iterative delayand-add procedure. This stimulus, also known as iterated rippled noise, is perceived as a noise-like stimulus with a certain tonal component caused by the spectral ripples introduced by the delay-and-add procedure (see e.g. [16]). The pitch of an acoustic signal is the perceptual correlate of its periodicity. The distance between the spectral ripples is the inverse of the delay time and determines the perceived pitch. As long as the delay for the RIS generation is $10 \mathrm{~ms}$ or more, the spectral ripples will not be resolved by the frequency analysis of the basilar membrane. The long-term spectral excitation pattern is therefore very similar for the random noise and the RIS stimuli. The pitch is related to time-interval processing in the auditory system rather than to spectral peaks [16].

Noise and RIS stimuli were chosen for this study rather than pure tones since the broadband excitation of the auditory system is known to evoke rather solid activation of auditory areas in the brain. In addition, an analysis of the contrast between random noise and RIS allows for an estimation to be made of the significance of time-interval processing in the canine auditory system, which has not been demonstrated before. The delay for the stimuli used throughout this study was between $20 \mathrm{~ms}$ and $10 \mathrm{~ms}$, corresponding to a periodicity pitch between 50 and $100 \mathrm{~Hz}$.

All sound stimuli were created in Matlab (The Mathworks Inc., Natick, MA, USA) and presented to the subjects binaurally at a sound level of $90 \mathrm{~dB}$ SPL using fMRI compatible headphones (Sensimetrics S14 insert headphones, Sensimetrics corp., Malden, USA) in combination with canine ear covers (Mutt Muffs, Safe and Sound Pets, Westminster, USA) for additional hearing protection. The stimuli were arranged alternatingly with periods of silence using two different paradigms: paradigm 1 consisted of Gaussian noise, regular interval sounds (RIS) and silence, each condition being repeated 40 times and presented in random order. Paradigm 2 involved eight periods of silence and eight periods of RIS, each period lasting $30 \mathrm{~s}$. Each stimulation paradigm was combined with a suitable imaging sequence (see below).

\section{Imaging}

Data acquisition was performed on a 3 Tesla Philips Achieva MRI scanner (Royal Philips, Amsterdam, the Netherlands) in combination with $11 \mathrm{~cm}$ diameter circular surface coils. Prior to the acquisition of the functional data, high-resolution anatomical images in the dorsal plane were obtained from each dog with a T1 weighted sequence with repetition time $(\mathrm{TR})=11 \mathrm{~ms}$ and echo time $(\mathrm{TE})=5.2 \mathrm{~ms}$ with a field of view $(\mathrm{FOV})$ of $220 \mathrm{~mm}$ and $0.7 \mathrm{~mm} \times 0.7 \mathrm{~mm} \times 0.7 \mathrm{~mm}$ isotropic voxels.

Afterwards, two different echoplanar T2* weighted sequences with a slice thickness of $2 \mathrm{~mm}$ for 20 contiguous slices in the dorsal plane, a FOV of $192 \mathrm{~mm}$, a matrix of $96 \times 96$ and an TE of $35 \mathrm{~ms}$ were used to acquire functional data sets. Sequence 1 used the sparse temporal sampling method [35], which is a wellestablished procedure for reducing the influence of the scanner noise on the obtained functional images in auditory fMRI-experiments. With this sequence, a volume of images was recorded every $10 \mathrm{~s}(\mathrm{TR}=10 \mathrm{~s})$, all images of one volume were recorded within a period of $3 \mathrm{~s}$, resulting in 7-s-gaps between the measurements in which stimuli could be presented unmasked by scanner noise (Fig. 5). With this sequence, 120 volumes were recorded in $20 \mathrm{~min}$.

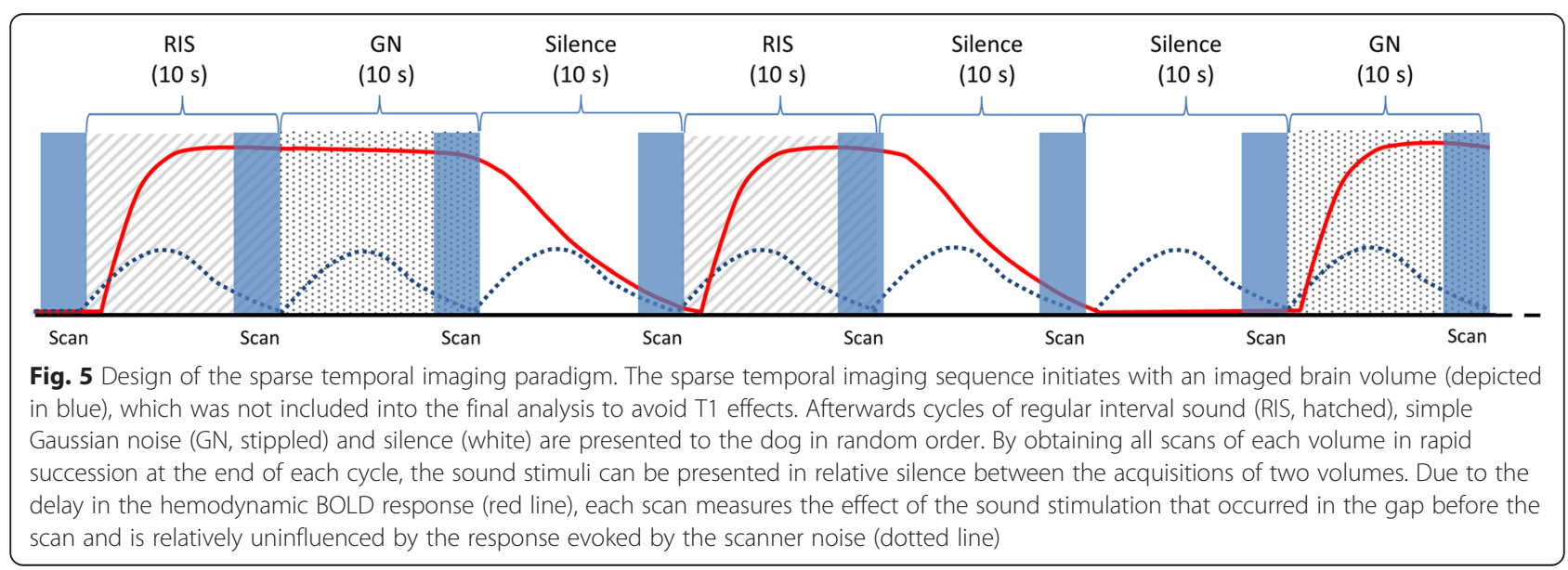


Sequence 2, which was combined with paradigm 2, was a conventional continuous sequence. With this sequence, 160 volumes were collected in $8 \mathrm{~min}(\mathrm{TR}=$ $3000 \mathrm{~ms}, \mathrm{TE}=35 \mathrm{~ms}$ ).

\section{Functional data analysis}

MRI data were processed and analyzed using SPM (FIL, Welcome Trust Center for Neuroimaging, UCL, London, England) in combination with the ROI-analysis toolbox MarsBar [54]. The two functional datasets acquired in each dog were analyzed separately. First, all functional images were realigned to the first image of the series. Then, a mean functional image was generated for each $\mathrm{dog}$, to which the anatomical image of the dog could be registered. For the single animal analysis, the following steps were performed without normalization of the functional data to a template image. Functional images were smoothed using a three-dimensional Gaussian filter ( $5 \mathrm{~mm}$ full width at half maximum). The general linear model was used with the standard hemodynamic response function provided by SPM as a reference to fit the measured time course of each voxel. Initially, all sound conditions combined were defined as the active condition in contrast to all periods in which no acoustic stimulus was presented as the rest condition. Afterwards, a $t$-test was used to test for differences between the two conditions. Following a recent auditory fMRI-study in anesthetized cats [21], the threshold for significant activation was set to $p<0.005$ (no multiple comparisons) and a cluster size of at least 3 adjacent voxels. Singer's 'The Brain of the Dog in Section' [42] and Pallazi's 'The Beagle Brain in Stereotaxic Coordinates' [55] were used to assign the activated areas to the underlying anatomical structures.

ROIs were defined for three different anatomical regions, which are known to exhibit a detectable BOLD response in fMRI-studies in humans [14]: 1. the caudal colliculi (CC), 2. the medial geniculate nuclei (MGN) and 3. the temporal cortex (TC). Three statistical values were calculated for each ROI for both functional sequences used: 1 . average $t$-value of all voxels included in the ROI, as a measure of significance of the difference found between active and rest conditions; 2 . the mean percentage signal change between these two conditions; 3. the number of activated voxels at $p$-value 0.005 as a percentage of the total number of voxels included in the ROI. Afterwards, an average value across all subjects was calculated for each statistical value for each sequence and each ROI to be later compared to the values obtained in the group analysis. Details concerning the configuration and placement of the ROIs and the statistical values calculated for each ROI can be found in [33].

For group analysis, the realigned functional images were normalized to Datta's brain atlas of the dog [34] before image smoothing. All subsequent steps of data preprocessing were performed similar to the single animal analysis. For statistical analysis, the data across all subjects were combined. Initially, the datasets acquired with the two functional sequences were analyzed separately. Afterwards, a combined analysis of the datasets was conducted. Like in the single subject analysis, all sound conditions were defined as the active condition in contrast to silence.

Finally, the influence of pitch on the elicited BOLD signal was examined by comparing the periods in which Gaussian noise stimuli (no pitch) were applied to periods with RIS-Stimulation (periodicity pitch). As the effect of periodicity pitch on the obtained BOLD signal was expected to be more feeble than the BOLD contrast in the comparison of sound vs. silence, the threshold for significant activation was reduced to $p=0.01$ and no cluster size-threshold was applied. The peak $t$-values of the activated voxels in each ROI were determined for this comparison. As no Gaussian noise stimuli were included in paradigm 2 , only data acquired with paradigm 1 in combination with imaging sequence 1 were used for this final step of data analysis.

\section{Data availability}

The data sets supporting the results of this article are available in the Dryad repository: doi:10.5061/ dryad.251h1.

\section{Abbreviations}

BOLD: blood oxygenation level dependent; CC: caudal colliculi; fMRI: functional magnetic resonance imaging; FOV: field of view; MGN: medial geniculate nuclei; RIS: regular interval sounds; ROI: region of interest; TC: temporal cortex; TE: echo time; TR: repetition time.

\section{Competing interests}

The authors hereby declare that they have no competing interests that might have influenced the content of the manuscript.

Authors' contributions

$\mathrm{ML}, \mathrm{PW}, \mathrm{HS}$ and IN conceived and designed the study; JPB and PD performed the experiments; JPB, ML and SU analyzed the data; all authors read, contributed to and approved the final manuscript.

\section{Acknowledgements}

We would like to thank Franziska Anders, Dr. Beate Laenger and Dr. Davina Ludwig for their advice and support in the experimental part of the study.

\section{Author details \\ ${ }^{1}$ Klinik für Kleintiere, Stiftung Tierärztliche Hochschule Hannover, Bünteweg 9, 30559 Hannover, Germany. ${ }^{2}$ Fachgebiet für Allgemeine Radiologie und Medizinische Physik, Stiftung Tierärztliche Hochschule Hannover, Bischofsholer Damm 15, 30173 Hannover, Germany. ${ }^{3}$ Medizinische Physik, Universität Oldenburg, 26111 Oldenburg, Germany.}

Received: 10 June 2015 Accepted: 4 December 2015

Published online: 20 February 2016

References

1. Strain GM. Canine deafness. Vet Clin North Am Small Anim Pract. 2012; 42(6):1209-24. 
2. McBrearty A, Penderis J. Transient evoked otoacoustic emissions testing for screening of sensorineural deafness in puppies. J Vet Intern Med. 2011;25(6):1366-71.

3. Penrod JP, Coulter DB. The diagnostic uses of impedance audiometry in the dog. J Am Anim Hosp Assoc. 1980;16:941-8.

4. Scheifele PM, Clark JG. Electrodiagnostic evaluation of auditory function in the dog. Vet Clin North Am Small Anim Pract. 2012;42(6):1241-57.

5. Sockalingam R, Filippich L, Sommerlad S, Murdoch B, Charles B. Transient-evoked and 2 F1-F2 distortion product oto-acoustic emissions in dogs: preliminary findings. Audiol Neurootol. 1998;3(6):373-85.

6. Strain GM. Congenital deafness and its recognition. Vet Clin North Am Small Anim Pract. 1999;29(4):895-907. vi.

7. Webb AA. Brainstem auditory evoked response (BAER) testing in animals. Can Vet J. 2009:50(3):313-8.

8. Wilson WJ, Mills PC. Brainstem auditory-evoked response in dogs. Am J Vet Res. 2005;66(12):2177-87

9. Strain GM. Brainstem Auditory Evoked Response (BAER). In: Deafness in Dogs and Cats. Cambridge: CABl; 2011.

10. Merzenich MM, Knight PL, Roth GL. Representation of cochlea within primary auditory cortex in the cat. J Neurophysiol. 1975;38(2):231-49.

11. Hall DA. fMRI of the Auditory Cortex. In: Faro SH, editor. Functional MRI: basic principles and clinical applications. New York: Springer; 2006.

12. Villringer A, Dirnagl U. Coupling of brain activity and cerebral blood flow: basis of functional neuroimaging. Cerebrovasc Brain Metab Rev. 1995;7(3):240-76.

13. Binder JR, Rao SM, Hammeke TA, Yetkin FZ, Jesmanowicz A, Bandettini PA Wong EC, Estkowski LD, Goldstein MD, Haughton VM, et al. Functional magnetic resonance imaging of human auditory cortex. Ann Neurol. 1994;35(6):662-72.

14. Kovacs S, Peeters R, Smits M, De Ridder D, Van Hecke P, Sunaert S. Activation of cortical and subcortical auditory structures at $3 T$ by means of a functional magnetic resonance imaging paradigm suitable for clinical use. Invest Radiol. 2006;41(2):87-96.

15. Talavage TM, Gonzalez-Castillo J, Scott SK. Auditory neuroimaging with fMRI and PET. Hear Res. 2014;307:4-15.

16. Patterson RD, Uppenkamp S, Johnsrude IS, Griffiths TD. The processing of temporal pitch and melody information in auditory cortex. Neuron. 2002; 36(4):767-76.

17. Plack CJ, Barker D, Hall DA. Pitch coding and pitch processing in the human brain. Hear Res. 2014;307:53-64

18. Cheung MM, Lau C, Zhou IY, Chan KC, Cheng JS, Zhang JW, Ho LC, Wu EX. BOLD fMRI investigation of the rat auditory pathway and tonotopic organization. Neuroimage. 2012;60(2):1205-11.

19. Voss HU, Salgado-Commissariat D, Helekar SA. Altered auditory BOLD response to conspecific birdsong in zebra finches with stuttered syllables. PLoS One. 2010;5(12):e14415.

20. Aguirre GK, Komaromy AM, Cideciyan AV, Brainard DH, Aleman TS, Roman AJ, Avants BB, Gee JC, Korczykowski M, Hauswirth WW, et al Canine and human visual cortex intact and responsive despite early retinal blindness from RPE65 mutation. PLoS Med. 2007;4(6):e230.

21. Brown TA, Joanisse MF, Gati JS, Hughes SM, Nixon PL, Menon RS, Lomber SG. Characterization of the blood-oxygen level-dependent (BOLD) response in cat auditory cortex using high-field fMR Neuroimage. 2013;64:458-65.

22. Chen G, Wang F, Dillenburger BC, Friedman RM, Chen LM, Gore JC, Avison MJ, Roe AW. Functional magnetic resonance imaging of awake monkeys: some approaches for improving imaging quality. Magn Reson Imaging. 2012;30(1):36-47.

23. Stefanacci L, Reber P, Costanza J, Wong E, Buxton R, Zola S, Squire L, Albright T. fMRI of monkey visual cortex. Neuron. 1998;20(6):1051-7.

24. Willis CK, Quinn RP, McDonell WM, Gati J, Parent J, Nicolle D. Functional MR as a tool to assess vision in dogs: the optimal anesthetic. Vet Ophthalmol. 2001;4(4):243-53.

25. Petkov Cl, Kayser C, Augath M, Logothetis NK. Optimizing the imaging of the monkey auditory cortex: sparse vs. continuous fMRI. Magn Reson Imaging. 2009;27(8):1065-73.

26. Lahti KM, Ferris CF, Li F, Sotak CH, King JA. Comparison of evoked cortical activity in conscious and propofol-anesthetized rats using functional MRI. Magn Reson Med. 1999;41(2):412-6.

27. Huettel SA, Song AW, McCarthy G. Functional magnetic resonance imaging vol. 1: Sinauer Associates Sunderland, MA; 2004.
28. Ashburner J, Friston K. Multimodal image coregistration and partitioning-a unified framework. Neuroimage. 1997:6(3):209-17.

29. Brett M, Johnsrude IS, Owen AM. The problem of functional localization in the human brain. Nat Rev Neurosci. 2002;3(3):243-9.

30. Crinion J, Ashburner J, Leff A, Brett M, Price C, Friston K. Spatial normalization of lesioned brains: performance evaluation and impact on fMRI analyses. Neuroimage. 2007;37(3):866-75.

31. Jiang D, Du Y, Cheng H, Jiang T, Fan Y. Groupwise spatial normalization of fMRI data based on multi-range functional connectivity patterns. Neuroimage. 2013;82:355-72.

32. Yost WA. Pitch of iterated rippled noise. J Acoust Soc Am. 1996:100(1):511-8.

33. Bach JP, Lupke M, Dziallas P, Wefstaedt P, Uppenkamp S, Seifert H, Nolte I. Functional magnetic resonance imaging of the ascending stages of the auditory system in dogs. BMC Vet Res. 2013;9:210.

34. Datta R, Lee J, Duda J, Avants BB, Vite CH, Tseng B, Gee JC, Aquirre GD, Aguirre GK. A digital atlas of the dog brain. PLoS One. 2012;7(12):e52140.

35. Hall DA, Haggard MP, Akeroyd MA, Palmer AR, Summerfield AQ, Elliott MR, Gurney EM, Bowtell RW. "Sparse" temporal sampling in auditory fMRI. Hum Brain Mapp. 1999;7(3):213-23.

36. Brazis P, Masdeu J, Biller J. Localization in Clinical Neurology. Alphen aan den Rijn, Netherlands: Wolters Kluwer; 2011.

37. Goldberg JM, Brown PB. Functional organization of the dog superior olivary complex: an anatomical and electrophysiological study. J Neurophysiol. 1968:31(4):639-56.

38. Heffner $\mathrm{H}$. Effect of auditory cortex ablation on localization and discrimination of brief sounds. J Neurophysiol. 1978:41(4):963-76.

39. Stepień I, Stepień L, Lubińska E. Function of dog's auditory cortex in tests involving auditory location cues and directional instrumental response. Acta Neurobiol Exp. 1989;50(1-2):1-12.

40. Andics A, Gacsi M, Farago T, Kis A, Miklosi A. Voice-sensitive regions in the dog and human brain are revealed by comparative fMRI. Curr Biol. 2014 24(5):574-8.

41. Hall AJ, Brown TA, Grahn JA, Gati JS, Nixon PL, Hughes SM, Menon RS, Lomber SG. There's more than one way to scan a cat: imaging cat auditory cortex with high-field fMRI using continuous or sparse sampling. J Neurosci Methods. 2014;224:96-106

42. Singer M. The Brain of the Dog in Section. W. B. Saunders Company, 1962. Plates 19-49, 62-88, 97-124.

43. Griffiths TD, Uppenkamp S, Johnsrude I, Josephs O, Patterson RD. Encoding of the temporal regularity of sound in the human brainstem. Nat Neurosci. 2001;4(6):633-7.

44. Walker KM, Bizley JK, King AJ, Schnupp JW. Cortical encoding of pitch: recent results and open questions. Hear Res. 2011;271(1-2):74-87.

45. Gelfer MP, Mikos VA. The relative contributions of speaking fundamental frequency and formant frequencies to gender identification based on isolated vowels. J Voice. 2005;19(4):544-54.

46. Smith DR, Patterson RD, Turner R, Kawahara $H$, Irino $T$. The processing and perception of size information in speech sounds. J Acoust Soc Am. 2005; 117(1):305-18.

47. Capranica RR. Vocal response of the bullfrog to natural and synthetic mating calls. J Acoust Soc Am. 1966;40(5):1131-9.

48. Koda H, Masataka N. A pattern of common acoustic modification by human mothers to gain attention of a child and by macaques of others in their group. Psychol Rep. 2002;91(2):421-2.

49. Kojima S, Izumi A, Ceugniet M. Identification of vocalizers by pant hoots, pant grunts and screams in a chimpanzee. Primates. 2003:44(3):225-30.

50. Nelson DA. Song frequency as a cue for recognition of species and individuals in the field sparrow (Spizella pusilla). J Comp Psychol. 1989; 103(2):171.

51. Berns GS, Brooks AM, Spivak M. Functional MRI in awake unrestrained dogs. PLoS One. 2012;7(5):e38027.

52. Berns GS, Brooks AM, Spivak M. Scent of the familiar: An fMRI study of canine brain responses to familiar and unfamiliar human and dog odors. Behav Processes. 2014; 110: 37-46.

53. Peelle JE, Eason RJ, Schmitter S, Schwarzbauer C, Davis MH. Evaluating an acoustically quiet EPI sequence for use in $\mathrm{fMRI}$ studies of speech and auditory processing. Neuroimage. 2010;52(4):1410-9.

54. Brett M, Valbregue R, Poline J-B. Region of interest analysis using the MarsBar toolbox for SPM 99. Neurolmage. 2002; 16:497.

55. Palazzi X. The Beagle Brain in Stereotaxic Coordinates.New York, USA Springer Science + Business Media; 2011. pp 67-77, 89-95 